TITLE:

\title{
Characterization of Coptis japonica CjABCB2, an ATP-binding cassette protein involved in alkaloid transport.
}

\section{AUTHOR(S):}

Shitan, Nobukazu; Dalmas, Fabien; Dan, Kazuyuki; Kato, Nobuhiko; Ueda, Kazumitsu; Sato, Fumihiko; Forestier, Cyrille; Yazaki, Kazufumi

\section{CITATION:}

Shitan, Nobukazu ...[et al]. Characterization of Coptis japonica CjABCB2, an ATP-binding cassette protein involved in alkaloid transport.. Phytochemistry 2013, 91: 109-116

\section{ISSUE DATE:}

2013-07

URL:

http://hdl.handle.net/2433/175501

\section{RIGHT:}

@ 2012 Elsevier Ltd.; この論文は出版社版でありません。引用の際には 出版社版をご確認ご利用ください。; This is not the published version. Please cite only the published version. 
Title:

Characterization of Coptis japonica $\mathrm{Cj} \mathrm{ABCB} 2$, an ATP-binding cassette protein involved in alkaloid transport

Authors:

Nobukazu Shitan ${ }^{\mathrm{a}, 1}$, Fabien Dalmas ${ }^{\mathrm{b}}$, Kazuyuki Dan ${ }^{\mathrm{c}}$, Nobuhiko Kato ${ }^{\mathrm{c}}$, Kazumitsu Ueda $^{\mathrm{d}, \mathrm{e}}$, Fumihiko Sato $^{\mathrm{c}}$, Cyrille Forestier $^{\mathrm{b}}$, and Kazufumi Yazaki ${ }^{\mathrm{a}}$ *

${ }^{a}$ Laboratory of Plant Gene Expression, Research Institute for Sustainable Humanosphere, Kyoto University, Gokasho, Uji 611-0011, Japan

${ }^{\mathrm{b}}$ Commissariat à l'Energie Atomique et aux Energies Alternatives (CEA), Direction of Life Sciences, Institute of Environmental Biology and Biotechnology, CEA Cadarache, UMR6191 CEA-CNRS-Aix-Marseille University, 13108 St Paul Lez Durance, France ${ }^{c}$ Division of Integrated Life Science, Graduate School of Biostudies, Kyoto University, Kyoto 606-8502, Japan

${ }^{\mathrm{d}}$ Division of Applied Life Sciences, Graduate School of Agriculture, Kyoto University, Kyoto 606-8502, Japan

${ }^{\mathrm{e}}$ Institute for Integrated Cell-Material Sciences (iCeMS), Kyoto University, Kyoto 606-8502, Japan

${ }^{1}$ Present address: Department of Natural Medicinal Chemistry, Kobe Pharmaceutical University, Motoyamakita-machi, Higashinada-ku, Kobe 658-8558, Japan 
*Corresponding author. Tel.: +81-774-38-3617; fax.: +81-774-38-3623. E-mail address: yazaki@ rish.kyoto-u.ac.jp (K. Yazaki). 


\section{Abstract}

Higher plants produce a large number of secondary metabolites. Among these are the alkaloids, a group of small nitrogen-containing molecules. Alkaloids often have strong biological activity that protects alkaloid-producing plants from herbivores, and often accumulate to high concentrations in a specific organelle of a particular organ in the producing plant. However, our knowledge of the membrane transport mechanism of alkaloids is still limited. Coptis japonica, a perennial ranunculaceous plant, produces the benzylisoquinoline alkaloid berberine. This alkaloid, though biosynthesized in the root tissues, accumulates in the rhizome, suggesting translocation of the molecule via xylem. In this study, we isolated a novel ATP-binding cassette (ABC) protein gene of B-type, Cjabcb2, from C. japonica. Northern analysis showed that $C j a b c b 2$ was preferentially expressed in the rhizome, which is the sink organ of berberine. Functional analysis of $\mathrm{Cj} A B C B 2$ using yeast suggested that $\mathrm{Cj} A B C B 2$ transports berberine in an inward direction. Membrane separation and in situ hybridization data indicated that CjABCB2 might be involved in the translocation of berberine from the root to the rhizome by transporting berberine at the plasma membrane of cells around the xylem of rhizome.

Keywords: ABC transporter, Alkaloid, Berberine, Coptis japonica, CjABCB2, Translocation 


\section{Introduction}

Higher plants produce a large number of alkaloids which have diverse chemical structures and biological activities. Some of them are used as medicines, such as anticancer drugs, analgesics, and antimalarial drugs (Croteau et al., 2000; Kutchan, 1995; Zenk and Juenger, 2007). Due to their strong antimicrobial and antifungal activities, as well as their other cytotoxicities, alkaloids in plants are presumed to be endogenous chemical barriers that protect the plants from pathogens or herbivores. For instance, berberine, a yellow benzylisoquinoline alkaloid, which is conventionally used as a bitter stomachic and an anti-diarrhetic in many countries, shows strong antimicrobial activity toward both Gram-positive and Gram-negative bacteria as well as other microorganisms.

It is often observed that a particular alkaloid preferentially accumulates in a particular organ in medicinal plants, rather than being equally distributed around the whole plant body (Maier et al., 1999). In some cases, alkaloids translocate from a source organ to a sink organ where they accumulate to a constant level. For example, nicotine is synthesized in root tissues in Nicotiana species, but mainly accumulates within the vacuoles of leaves (Hashimoto Yamada 1994). Moreover, the movement of biosynthetic intermediates of alkaloids from a specific cell layer to other cell layers has also been suggested (Facchini and De Luca, 2008; Kutchan, 2005; Weid et al., 2004). Details of the movement of alkaloids within the organelles in a cell are summarized elsewhere (Shitan and Yazaki, 2007). Membrane transport of alkaloids is a key biological event that drives movement of alkaloids among compartments, but the mechanisms of membrane transport, as well as the accumulation machinery of alkaloids in plants, has remained largely unknown despite several decades of research. 
A simple model for alkaloid accumulation in plant vacuoles is the ion-trap mechanism (Matile, 1976). In this model, alkaloids, which are in a lipophilic state in the neutral $\mathrm{pH}$ of cytosol (ca. $\mathrm{pH}$ 7.2), can freely pass through the tonoplast by simple diffusion. In the acidic condition of the vacuolar lumen (e.g. pH 5.5), however, they are immediately protonated to form a hydrophilic ion, which is incapable of permeating through the membrane, resulting in the trapping of alkaloid molecule inside. In this model, alkaloid movement takes place by one-way diffusion, in which transporter molecules are not necessary. Contrary to this model, studies by Dr. Zenk's group and also by other independent research groups have demonstrated that the vacuolar transport of alkaloids is managed by specific carriers that can recognize stereochemistry of transport substrates in an energy-requiring manner (Deus-Neumann and Zenk, 1984, 1986; Mende and Wink, 1987). These reports suggest the involvement of a proton-antiport carrier system. A third mechanism proposed for vacuolar transport of alkaloids is via a directly energized transporter, the ATP-binding cassette (ABC) transporter, which might also be responsible for the transport of other secondary metabolites (Klein et al., 2000). Recent progress in this field has identified several transporters for alkaloids. For instance, Nt-JAT1 (Nicotiana tabacum jasmonate-inducible alkaloid transporter1) and NtMATE1, belonging to the MATE (multidrug and toxic compound extrusion) transporter family, are involved in vacuolar transport of alkaloids in N. tabacum (Morita et al., 2009; Shoji et al., 2009 ). For the vacuolar accumulation of alkaloid, a vesicle-mediated mechanism has also been proposed (Amann et al., 1986; Bock et al., 2002; Galneder et al., 1988). However, our knowledge of the number and type of transporters responsible for the transport and translocation of the many divergent alkaloids in plants is still very limited. Hence, 
further identification and characterization of alkaloid transporters is of critical importance for progress in the field.

In this study, we have used Coptis japonica (Ranunculaceae) as a model plant to isolate transporters for endogenous alkaloids. $C$. japonica is a perennial medicinal plant grown in Asian countries, and produces berberine as its main alkaloid. Berberine accumulates to high levels in the rhizome of $C$. japonica, while genes coding for the biosynthetic enzymes of berberine are specifically expressed in the root tissues (Ikezawa et al., 2003), suggesting that berberine translocates from the root to the rhizome via transporter molecules. A cell culture system of $C$. japonica that efficiently produces and accumulates berberine has been maintained for many years. It has been demonstrated in cultured cells that berberine exclusively accumulates in the vacuoles (Sato et al., 1992). The $C$. japonica cell cultures can also actively take up berberine exogenously added to the culture medium, and the administered berberine is also sequestrated in the vacuolar lumen (Sakai et al., 2002). The cell culture system of $C$. japonica is thus a powerful tool for the biochemical analysis of alkaloid transport and is a suitable source for isolation of transporter cDNAs. Our previous study showed that the uptake of berberine by $C$. japonica cells depends on the endogenous ATP level, and several inhibitors of human ABCB1 (MDR1), a representative ABC transporter responsible for multidrug resistance of cancer cells, significantly suppresses berberine uptake from the medium (Sakai et al., 2002). We further clarified that a B-type ABC protein $\mathrm{CjMDR} 1$, which is now called $\mathrm{Cj} \mathrm{ABCB} 1$ according to the unified nomenclature (Verrier et al., 2008), functions as a berberine transporter at the plasma membrane (Shitan et al., 2003). 
In this paper, we have isolated two cDNAs for novel $\mathrm{ABCB}$ proteins, $\mathrm{Cj} \mathrm{ABCB} 2$ and 3, which have significant amino acid similarities with $\mathrm{Cj} A B C B 1$, from cultured cells of $C$. japonica. We have mainly focused on the characterization of Cjabcb2, and demonstrated that $\mathrm{Cj} \mathrm{ABCB} 2$ may be mediating the translocation of berberine from root to the rhizome in plant. 


\section{Results}

\subsection{Cloning and Sequence Analysis of $\mathrm{Cjabcb2}$ and $\mathrm{Cjabcb} 3$}

Our previous study suggested that an ABC protein of B-type was involved in berberine uptake in cultured $C$. japonica cells (Sakai et al., 2002). To obtain relevant genes encoding $\mathrm{ABCB}$ proteins, degenerate primers corresponding to the highly conserved amino acid sequences for nucleotide binding folds (NBFs) of ABC proteins were designed. By a combination of RT-PCR, 5' and 3' RACE, we obtained two full-length cDNAs of ABCB proteins, which were designated Cjabcb2 and 3 (Coptis japonica $a b c b$ ). Both $C j a b c b 2$ and 3 were ca. $4.2 \mathrm{~kb}$ in length and coded for putative polypeptides composed of 1,292 and 1,288 amino acids, respectively. Hydropathy analysis with the program on the 'DAS' transmembrane prediction server (http://www.sbc.su.se/ miklos/DAS/) suggested that the topologies of CjABCB2 and 3 are very similar to that of human $\mathrm{ABCB}$ 1, i.e. twelve transmembrane helices and two NBFs representing full-size $\mathrm{ABC}$ proteins. In the phylogenetic relationship of B-type plant $\mathrm{ABC}$ transporters, both $\mathrm{CjABCB} 2$ and 3 belong to Clade II (Fig. 1) (Geisler and Murphy, 2006). In this clade, two ABC proteins have already been characterized; $\mathrm{Cj} \mathrm{ABCB} 1$ of $C$. japonica functions as a berberine influx transporter and AtABCB4 (AtPGP4) is involved in auxin transport in Arabidopsis root (Cho et al., 2007; Santelia et al., 2005; Shitan et al., 2003; Terasaka et al., 2005). CjABCB2 showed strong amino acid sequence similarities to $\mathrm{Cj} \mathrm{ABCB} 1(82 \%)$ and AtABCB4 (70\%), whereas CjABCB3 showed lower similarity to CjABCB1 (61\%) and AtABCB4 (59\%) than CjABCB2.

2.2. Cjabcb2 and $\mathrm{Cjabcb} 3$ expression in intact plant and cultured cells of $\mathrm{C}$. japonica 
The expression of $C j a b c b 2$ and 3 in the cultured $C$. japonica cells and various organs of intact plants were analyzed by northern blot hybridization. In the cultured $C$. japonica cells, the expression level of Cjabcb2 remained almost constant (Fig. 2A), as did the berberine content in the cells (Fig. 2B), while the mRNA level appeared to be slightly higher in the growth phase than in the stationary and lag phase. By contrast, Cjabcb3 expression in cultured cells gradually increased in the growth phase up to 14 days after inoculation and then decreased (Fig. 2A).

In intact plants, a clear, tissue-specific preference of $C j a b c b 2$ expression was observed. Higher expression of Cjabcb2 mRNA was detected in rhizome, the main accumulation site of berberine (Ikezawa et al., 2003) (Fig. 3), whereas almost no detectable transcripts were seen in other organs. These results reveal a clear correlation between the gene expression of Cjabcb2 and the accumulation of the alkaloid in plant organs. Cjabcb3 expression was, however, almost undetectable in intact plants, suggesting that the contribution of this gene to the specific berberine accumulation in rhizome is not large. Thus, further analysis was performed only with $C j a b c b 2$.

\subsection{CjABCB2 functions as a berberine influx pump}

Because Cjabcb2 expression showed a correlation to the berberine accumulation in intact plant, we investigated whether $\mathrm{Cj} \mathrm{ABCB} 2$ had the ability to transport berberine. To characterize the transport function of $\mathrm{Cj} \mathrm{ABCB} 2$, we used Saccharomyces cerevisiae AD12345678 strain, which shows high drug sensitivity due to the deletion of several ABC protein-related genes (Decottignies et al., 1998). An expression construct of CjABCB2 downstream of the strong constitutive promoter PMA1 was introduced into the yeast strain. Since berberine is cytotoxic to many unicellular organisms like bacteria 
and yeast, we analyzed the function of $\mathrm{Cj} A B C B 2$ by estimating yeast growth in the presence of berberine and several other drugs for comparison (Fig. 4). CjABCB2 transformant appeared to be more sensitive to berberine, although the difference was small. A larger difference in drug sensitivity between CjABCB2 transformant and the empty vector control was found with 4-nitroquinoline $N$-oxide (4-NQO), which has quinoline moiety in its structure and a substrate of yeast pleiotropic drug resistance (PDR)-type ABC protein, SNQ2 (Servos et al., 1993). These data suggested that 4-NQO was recognized as a substrate of $\mathrm{Cj} \mathrm{ABCB} 2$ and taken up into cells, resulting in increased sensitivity to 4-NQO in yeast. However, cycloheximide, a substrate of another yeast $\mathrm{ABC}$ protein PDR5, showed no clear difference between $\mathrm{Cj} A B C B 2$-expressing and control yeast cells, which indicated that substrates of $\mathrm{Cj} \mathrm{ABCB} 2$ were not as broad as those of yeast PDR5 or human ABCB1. From the structure similarity of berberine and 4-NQO, it is presumed that isoquinoline or quinoline derivatives are preferable substrates for $\mathrm{Cj} \mathrm{ABCB} 2$.

\subsection{CjABCB2 was localized at the plasma membrane}

To investigate the subcellular localization of $\mathrm{CjABCB} 2$ in $C$. japonica cells, we prepared polyclonal antibodies against a peptide sequence of $\mathrm{Cj} A B C B 2$. These antibodies reacted with a $140 \mathrm{kDa}$ protein in yeast cells expressing CjABCB2, indicating that these antibodies recognized CjABCB2 (Fig. 5A). For subcellular localization analysis of $\mathrm{Cj} A B C B 2$, microsomal membranes were first fractionated by sucrose density gradient centrifugation and then a western blot was performed. As shown in Fig. 5B, CjABCB2 co-fractionated with the plasma membrane $\mathrm{H}^{+}$-ATPase, peaking at $30 / 40 \%$ sucrose fraction. In contrast, vacuolar $\mathrm{H}^{+}$-pyrophosphatase 
(V-PPase), a marker of tonoplast, peaked at the 0/20\% sucrose fraction, and membranes containing luminal binding protein (BiP), a marker of endoplasmic reticulum (ER), formed sediment at positions different from $\mathrm{Cj} A B C B 2$. These results suggested that CjABCB2 was associated with the plasma membrane and not with the tonoplast or ER.

The aqueous two-phase partitioning method was also performed to confirm the plasma membrane localization of $\mathrm{CjABCB} 2$ (Fig. 5C). CjABCB2 and $\mathrm{H}^{+}$-ATPase were highly enriched in the upper-phase fraction (U) and greatly depleted in the lower-phase fraction (L). V-PPase, tonoplast marker, was enriched in the lower-phase fraction as logically expected. Bip, ER marker, was partly detected in the upper-phase fraction, but was also detected in the lower-phase, unlike $\mathrm{H}^{+}$-ATPase. Taken together, these results indicated that $\mathrm{Cj} \mathrm{ABCB} 2$ was localized at the plasma membrane.

\subsection{In situ localization of Cjabcb2 mRNA}

To identify the physiological role of $\mathrm{Cj} A B C B 2$ in planta, in situ hybridization was performed using an antisense RNA probe prepared from $C j a b c b 2$ cDNA. We analyzed two organs, rhizome and root, corresponding to the sink organ for berberine accumulation and the source organ for its biosynthesis, respectively. In the rhizome section, weak signals for the Cjabcb2 mRNA were observed with the antisense probe around the vascular tissue, particularly xylem cells (Fig. 6A), whereas no signal was observed with the sense probe as a negative control (Fig. 6B). Almost no signals were detected in root sections with either antisense or sense probe (Fig. $6 \mathrm{C}$ and D). Berberine may translocate from the root to the rhizome via several transporters, i.e., efflux from source cells and uptake at sink cells both at plasma and vacuolar membranes, 
and these results suggest that an $\mathrm{ABC}$ transporter, $\mathrm{Cj} \mathrm{ABCB} 2$, may participate in the accumulation of berberine in rhizome by acting as the berberine influx transporter. 


\section{Discussion}

Recently there have been reports of the involvement of $\mathrm{ABC}$ proteins in the transport of secondary metabolites; these reports are summarized in review articles (Yazaki, 2005, 2006). The maize ABCC (multidrug resistance associated protein, MRP)-type transporter, ZmMRP3 was required for the vacuolar accumulation of anthocyanin (Goodman et al., 2004). For plant pathogen resistance, Nicotiana plumbaginifolia $\mathrm{ABCG}$ (PDR)-type $\mathrm{ABC}$ transporter, NpPDR1 plays an important role by excreting sclareol, an antifungal diterpenoid compound in Nicotiana species, to the leaf surface (Jasinski et al., 2001). Wax is a complex mixture of lipophilic metabolites, which protect plants from drought stress, and half-size ABC proteins, AtABCG11 (AtWBC11) and AtABCG12 (AtWBC12), ABCG (white-brown complex, WBC)-type proteins, are involved in the secretion of wax components on the plant surface (Bird et al., 2007; Luo et al., 2007; Pighin et al., 2004; Ukitsu et al., 2007). These findings suggest that a number of $\mathrm{ABC}$ transporters are involved in the membrane transport of endogenous metabolites in plant body.

We isolated in this study two cDNAs, Cjabcb2 and Cjabcb3, which belong to $\mathrm{ABC}$ protein of B-type. Functional and expression analyses suggested that $\mathrm{Cj} A B C B 2$ was involved in the unloading of berberine in the rhizome, i.e., berberine is biosynthesized in root tissues and translocates upward, probably via xylem transport, whereas in the rhizome the berberine is trapped by plasma membrane-localized CjABCB2 and accumulates in rhizome tissue. Since CjABCB1, the closest member of CjABCB2, was shown to play a similar function in berberine transport (Shitan et al., 2003), these two ABC proteins may function in a cooperative manner for berberine accumulation in rhizome. Since the rhizome is an important sink organ for starch as 
well as berberine in the $C$. japonica plant, the accumulation of berberine in rhizome has a clear benefit, as the anti-microbial alkaloid, acting as a chemical defense, can protect the carbohydrate-rich sink tissue from attack by soil pathogens. The expression of $\mathrm{CjABCB} 1$ is, however, observed not only in rhizome but also in petiole, peduncle, and flower (Yazaki et al., 2001). By contrast CjABCB2 is expressed only in rhizome (Fig. 3), suggesting that $\mathrm{Cj} A B C B 2$ is specialized for berberine accumulation in rhizome, whereas $\mathrm{Cj} \mathrm{ABCB} 1$ might be responsible for the berberine accumulation in other tissues of low berberine content.

For characterization of plant $\mathrm{ABC}$ proteins, selection of a suitable expression system is crucial. In the case of $\mathrm{CjABCB} 2$, although we first tried to express it in Xenopus oocyte, we did not observe accumulation of the protein in this heterologous host (data not shown). These data suggested that CjABCB2 was not compatible with Xenopus oocyte, even though it has high amino acid identity (82\%) with CjABCB1, whose expression has been successfully performed in oocytes (Shitan et al., 2003). As a second choice of heterologous expression, we selected a yeast system and were able to characterize $\mathrm{Cj} \mathrm{ABCB} 2$ function. Other plant $\mathrm{ABCB}$ proteins, AtABCB1 (AtPGP1) and AtABCB4 (AtPGP4) were expressed and characterized in Hela cells, yeast cells, and BY-2 cells, to demonstrate the transport function of auxin (Cho et al., 2007; Geisler et al., 2005; Santelia et al., 2005; Terasaka et al., 2005). Yeast vesicle transport analyses were demonstrated for ABCC members as well; of these members, AtABCC1 (AtMRP1), 2 and 3 had transport activity for glutathione conjugate (Lu et al., 1998; Tommasini et al., 1998), and AtABCC5 (AtMRP5) could transport estradiol-17-( $\beta$-D-glucuronide) (Gaedeke et al., 2001). Sulfonylurea-binding activity of AtABCC5 was shown by HEK293 cell expression system (Lee et al., 2004). Other ABC 
proteins were characterized directly in mutant plants, in which $\mathrm{ABC}$ protein expression was modified by T-DNA insertion, antisense or overexpression (Goodman et al., 2004; Pighin et al., 2004). In general, however, few plant ABC proteins have been biochemically characterized, probably due to the difficulty in finding an appropriate heterologous host. To characterize $\mathrm{ABC}$ protein function, it would be very important to screen several heterologous expression systems.

The final intracellular accumulation site of berberine is vacuole in $C$. japonica. For vacuolar alkaloid accumulation, Dr. Zenk's group predicted a highly specific carrier-mediated and energy-dependent proton antiport system as early as the 1980s. (Deus-Neumann and Zenk, 1984, 1986). Our biochemical analysis in C. japonica suggested that vacuolar berberine transport is indeed mediated by an $\mathrm{H}^{+}$/berberine antiporter (Otani et al., 2005). As an $\mathrm{H}^{+} /$substrate antiporter family, MATE-type transporters have recently been reported to transport various secondary metabolites, which were reviewed previously (Yazaki et al., 2008). We have shown that Nt-JAT1, a tobacco MATE transporter, is involved in nicotine translocation and vacuolar accumulation in tobacco leaves (Morita et al., 2009). In fact, though it is known that MATE-type transporters, functioning as $\mathrm{H}^{+}$/substrate antiporters, serve an important membrane protein family responsible for transporting various endogenous metabolites (Gomez et al., 2009; Marinova et al., 2007; Maron et al., 2010), it is still unknown whether or not the vacuolar berberine transporter is a member of the MATE-type proteins. Further studies on vacuolar transporters in $C$. japonica are necessary in order to understand the system responsible for transport and sequestration of berberine in vacuoles of the cells of this plant. 


\section{Conclusions}

We isolated a novel $\mathrm{ABC}$ protein, $\mathrm{Cj} \mathrm{ABCB} 2$, from the berberine-producing plant C. japonica. $\mathrm{Cj} \mathrm{ABCB} 2$ was specifically expressed in the rhizome, where berberine accumulates to high levels. This protein localized to the plasma membrane and functioned as a berberine influx transporter. In situ hybridization showed that Cjabcb2 was expressed specifically around xylem tissue of rhizome. These data suggested that $\mathrm{Cj} A B C B 2$ was involved in the berberine translocation by unloading the protein in the rhizome. Transport of secondary metabolites including alkaloids is a hot topic of late (Shitan and Yazaki, 2007; Yazaki, 2005; Yazaki et al., 2009), and the present findings may contribute at least partly to an understanding of the molecular mechanism of alkaloid transport in plants. 


\section{Experimental}

\subsection{Cultured Cells}

High berberine-producing cultured $C$. japonica cells, which were originally induced from rootlets of $C$. japonica Makino var. dissecta (Yamabe) Nakai, were maintained as described previously (Sato and Yamada, 1984).

\subsection{Chemicals}

Berberine and other chemicals used in this study were purchased from Wako Pure Chemicals (Osaka, Japan) or Nakalai Tesque (Kyoto, Japan).

\subsection{Isolation of full-length abcb-like gene from cultured $\mathrm{C}$. japonica cells}

Total RNA prepared from $C$. japonica cells using RNeasy Plant Mini Kit (Qiagen) was reverse-transcribed with M-MLV reverse transcriptase (NEB). Nested PCR was carried out with Taq DNA polymerase (Takara), using the DNA-RNA hybrid as a template, and two sets of degenerate primers designed from the highly conserved amino acid sequences in the nucleotide-binding fold (NBF) which is the domain responsible for the ATP-binding property of ABC transporters; Fwd-primer1, 5'-MARACIYTIGCHYTIGTIGG-3' (n- (Q/K)TTLALVG -c) and Rv-primer1, 5'-GCRCTXHTXGCYTCRTCXARXAG-3' (n- LLDEATSA -c): Fwd-primer2, 5'-GGITGYGGIAARWSIWSIGT-3' (n- GCGKS(T/S)V -c) and Rv-primer2, 5'-GCDATXCKYTGYTTYTGXCCXCC-3' (n- GGQKQRIA -c). As the PCR products, two $320 \mathrm{bp}$ fragments for the NBF domain of ABCB-type transporter were isolated. Using two specific primers corresponding to the internal sequences of these DNA fragments, RT-PCR was performed again using a heat-stable DNA polymerase of 
high-fidelity KOD (TOYOBO), resulting in the amplification of a DNA fragment (2.2 kb) which was significantly similar to known ABCB-like transporters. To obtain full-length cDNA clones, 5' and 3' RACE using a cDNA amplification kit (Clontech) were used. The accession numbers of $C j a b c b 2$ and $C j a b c b 3$ are AB674325 and AB674326, respectively.

\subsection{Organ-specific expression}

Total RNAs were isolated from root, rhizome, petiole, leaf, peduncle, bud, flower, and seed of the intact plant of $C$. japonica. Aliquots of $10 \mu \mathrm{g}$ of RNA were loaded on formamide-containing agarose gel, electrophoresised and blotted onto a nylon membrane. As the hybridization probe, 3'-UTR of Cjabcb2 or Cjabcb3 (200 bp) were used. As the load control, 18S rRNA was used.

\subsection{Time-course of gene expression and berberine contents}

Time-course of Cjabcb2 or Cjabcb3 expression in cultured cells of Coptis japonica was analyzed by northern hybridization. The cultured cells were harvested 2, 5, 9, 14, 21 days after inoculation, from which poly $(\mathrm{A})^{+}$RNA was isolated and loaded on formamide-containing agarose gel. As the hybridization probe, 3'-UTR of Cjabcb2 or Cjabcb3 (200 bp) were used. ATP synthase ( $\beta$ subunit) was used to analyze the loading amount. Cell growth was monitored and berberine content in the cells was quantified by HPLC analysis. For HPLC analysis, alkaloids were extracted from cells with HCl-acidified methanol until the cells lost their yellow color. The supernatant was subjected to HPLC analysis: mobile phase, $50 \mathrm{mM}$ tartaric acid solution containing 10 mM SDS - acetonitrile - methanol (100: $100: 25)$; column, TSK-GEL ODS-80TM 
(TOSOH, 4.6 i.d. X $250 \mathrm{~mm}$ ); temperature, $40^{\circ} \mathrm{C}$; flow rate, $1.2 \mathrm{ml} / \mathrm{min}$; detection, absorbance measured at $260 \mathrm{~nm}$.

\subsection{Peptide antibody against CjABCB2}

A KLH (keyhole limpet hemocyanin) conjugate of an oligopeptide of CjABCB2, at position 665 (n-CSRGSSGVGNSSRKS -c), was injected into rabbit according to the standard protocol (Kurabo Industries, Japan). After the fourth boost, antiserum was recovered and used for immunoblot analysis without further purification.

\subsection{Functional analysis of $C j A B C B 2$ using Yeast}

Cjabcb2 cDNA (4.0 kb) was subcloned into yeast expression vector pDR196, kindly provided by Dr. W. Frommer (Carnegie Institution, Stanford, USA), at the Not I site. The resulting plasmid, pDR-CjABCB2, was used to transform strain AD12345678

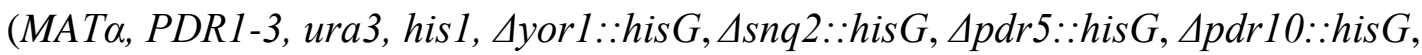
Apdr11::hisG, $\Delta y c f 1:: h i s G, \Delta p d r 3: \because h i s G$; and $\Delta p d r 15:: h i s G)$ (Decottignies et al., 1998) by the lithium acetate method (Yazaki et al., 2002), and then selected by SD medium (-uracil). The drug sensitivity of yeast transformants was tested by spotting SD- cultures (-uracil) onto agar plates containing various compounds. Five $\mu 1$ of the transformant diluted to same density $\mathrm{A}_{600}=0.5$ was spotted onto each plate and growth was monitored after incubation of the cells for $44 \mathrm{~h}$ at $25^{\circ} \mathrm{C}$. The drugs, berberine and cycloheximide, used in this study were dissolved in water, while 4-nitroquinoline $\mathrm{N}$-oxide was dissolved in acetone.

\subsection{Isolation of Plasma Membranes}


Plasma membranes were purified from a microsomal fraction of $C$.japonica cells by partitioning in an aqueous polymer two-phase system and sucrose gradient fractionation as described previously (Larsson et al., 1987; Otani et al., 2005). Protein electrophoreses and electrophoretic transfer was performed as described previously (Shitan et al., 2003). Antibodies used for immunodetection were against CjABCB2, vacuolar $\mathrm{H}^{+}$-pyrophosphatase from Arabidopsis (V-PPase), endoplasmic reticulum BiP (BiP), and plasma membrane $\mathrm{H}^{+}$-ATPase $\left(\mathrm{H}^{+}\right.$-ATPase).

\subsection{In Situ hybridization}

Root and rhizome were fixed on ice in freshly prepared solutions of 2 and $4 \%$ formaldehyde in 50\% ethanol and 10\% acetic acid, respectively, for $3 \mathrm{~h}$. The samples were dehydrated through an ethanol series, and embedded with Paraplast plus (OXFORD). Sections 10- to $20-\mu \mathrm{m}$ thick prepared with a microtome (RM 2155, Leica) were hybridized with the digoxigenin-labeled antisense or sense RNA probe of Cjabcb2, which is a 178-bp fragment containing the 3'-untranslated region. In situ hybridization and the detection procedures were performed as described previously (Yamada et al., 1995). 


\section{Acknowledgements}

We thank Dr. W. Frommer (Carnegie Institution, Stanford, CA, USA) for the gift of pDR196; Dr. M. A. De Waard (Wageningen University, The Netherlands) for providing the yeast AD12345678 strain; Dr. M. Boutry (Université Catholique de Louvain, Belgium) for anti- $\mathrm{H}^{+}$-ATPase antibodies; Dr. N. Koizumi (Osaka Prefecture University, Japan) for anti-BiP antibodies; Dr. M. H. Sato (Kyoto Prefectural University, Japan) for anti-V-PPase antibodies; and Kyoto Botanical Garden of Takeda Chemical Industries for the intact plants of $C$. japonica. Analysis of DNA sequencing was conducted with the Life Research Support Center in the Akita Prefectural University. This work was supported by a Grant-in-Aid for Scientific Research 19039019 (to K.Y.), the Uehara Foundation (to K.Y.), a research fellowship from the Japan Society for the Promotion of Science (grant No. 160405 to N.S.), grant from Inoue Foundation for Science, and Short-term Research Grant from Toyobo Biofoundation. 


\section{References}

Amann, M., Wanner, G., Zenk, M. H., 1986. Intracellular compartmentation of two enzymes of berberine biosynthesis in plant cell cultures. Planta 167, 310-320.

Bird, D., Beisson, F., Brigham, A., Shin, J., Greer, S., Jetter, R., Kunst, L., Wu, X., Yephremov, A., Samuels, L., 2007. Characterization of Arabidopsis

ABCG11/WBC11, an ATP binding cassette (ABC) transporter that is required for cuticular lipid secretion. Plant J 52, 485-498.

Bock, A., Wanner, G., Zenk, M. H., 2002. Immunocytological localization of two enzymes involved in berberine biosynthesis. Planta 216, 57-63.

Cho, M., Lee, S. H., Cho, H. T., 2007. P-glycoprotein4 displays auxin efflux transporter-like action in Arabidopsis root hair cells and tobacco cells. Plant Cell 19, 3930-3943.

Croteau, R., Kutchan, T. M., Lewis, N. G., 2000. Natural Products (Secondary Metabolites). Biochemistry \& Molecular Biology of Plants (Buchanan, B., Gruissem, W. and Jones, R., Eds.), American Society of Plant Physiologists, Maryland., pp. 1250-1318.

Decottignies, A., Grant, A. M., Nichols, J. W., de Wet, H., McIntosh, D. B., Goffeau, A., 1998. ATPase and multidrug transport activities of the overexpressed yeast ABC protein Yor1p. J Biol Chem 273, 12612-12622.

Deus-Neumann, B., Zenk, M. H., 1984. A highly selective alkaloid uptake system in vacuoles of higher plants. Planta 162, 250-260.

Deus-Neumann, B., Zenk, M. H., 1986. Accumulation of alkaloids in plant vacuoles does not involve an ion-trap mechanism. Planta 167, 44-53.

Facchini, P. J., De Luca, V., 2008. Opium poppy and Madagascar periwinkle: model non-model systems to investigate alkaloid biosynthesis in plants. Plant J 54, 763-784.

Gaedeke, N., Klein, M., Kolukisaoglu, U., Forestier, C., Muller, A., Ansorge, M., Becker, D., Mamnun, Y., Kuchler, K., Schulz, B., Mueller-Roeber, B., Martinoia, E., 2001. The Arabidopsis thaliana ABC transporter AtMRP5 controls root development and stomata movement. Embo J 20, 1875-1887.

Galneder, E., Rueffer, M., Wanner, G., Tabata, M., Zenk, M. H., 1988. Alternative final steps in berberine biosynthesis in Coptis japonica cell cultures. Plant Cell Reports 7, 1-4.

Geisler, M., Blakeslee, J. J., Bouchard, R., Lee, O. R., Vincenzetti, V., Bandyopadhyay, A., Titapiwatanakun, B., Peer, W. A., Bailly, A., Richards, E. L., Ejendal, K. F., Smith, A. P., Baroux, C., Grossniklaus, U., Muller, A., Hrycyna, C. A., Dudler, R., 
Murphy, A. S., Martinoia, E., 2005. Cellular efflux of auxin catalyzed by the Arabidopsis MDR/PGP transporter AtPGP1. Plant J 44, 179-194.

Geisler, M., Murphy, A. S., 2006. The ABC of auxin transport: The role of p-glycoproteins in plant development. FEBS Lett 580, 1094-1102.

Gomez, C., Terrier, N., Torregrosa, L., Vialet, S., Fournier-Level, A., Verries, C., Souquet, J. M., Mazauric, J. P., Klein, M., Cheynier, V., Ageorges, A., 2009. Grapevine MATE-type proteins act as vacuolar H+-dependent acylated anthocyanin transporters. Plant Physiol 150, 402-415.

Goodman, C. D., Casati, P., Walbot, V., 2004. A multidrug resistance-associated protein involved in anthocyanin transport in Zea mays. Plant Cell 16, 1812-1826.

Ikezawa, N., Tanaka, M., Nagayoshi, M., Shinkyo, R., Sakaki, T., Inouye, K., Sato, F., 2003. Molecular cloning and characterization of CYP719, a methylenedioxy bridge-forming enzyme that belongs to a novel P450 family, from cultured Coptis japonica cells. J Biol Chem 278, 38557-38565.

Jasinski, M., Stukkens, Y., Degand, H., Purnelle, B., Marchand-Brynaert, J., Boutry, M., 2001. A plant plasma membrane ATP binding cassette-type transporter is involved in antifungal terpenoid secretion. Plant Cell 13, 1095-1107.

Klein, M., Martinoia, E., Hoffmann-Thoma, G., Weissenbock, G., 2000. A membrane-potential dependent ABC-like transporter mediates the vacuolar uptake of rye flavone glucuronides: regulation of glucuronide uptake by glutathione and its conjugates. Plant J 21, 289-304.

Kutchan, T. M., 1995. Alkaloid Biosynthesis - The Basis for Metabolic Engineering of Medicinal Plants. Plant Cell 7, 1059-1070.

Kutchan, T. M., 2005. A role for intra- and intercellular translocation in natural product biosynthesis. Curr Opin Plant Biol 8, 292-300.

Larsson, C., Widell, S., Kjellbom, P., 1987. Preparation of high-purity plasma membranes. Methods Enzymology 148, 558-568.

Lee, E. K., Kwon, M., Ko, J. H., Yi, H., Hwang, M. G., Chang, S., Cho, M. H., 2004. Binding of sulfonylurea by AtMRP5, an Arabidopsis multidrug resistance-related protein that functions in salt tolerance. Plant Physiol 134, 528-538.

Lu, Y. P., Li, Z. S., Drozdowicz, Y. M., Hortensteiner, S., Martinoia, E., Rea, P. A., 1998. AtMRP2, an Arabidopsis ATP binding cassette transporter able to transport glutathione S-conjugates and chlorophyll catabolites: functional comparisons with Atmrp1. Plant Cell 10, 267-282. 
Luo, B., Xue, X. Y., Hu, W. L., Wang, L. J., Chen, X. Y., 2007. An ABC transporter gene of Arabidopsis thaliana, AtWBC11, is involved in cuticle development and prevention of organ fusion. Plant Cell Physiol 48, 1790-1802.

Maier, U. H., Rodl, W., Deus-Neumann, B., Zenk, M. H., 1999. Biosynthesis of Erythrina alkaloids in Erythrina crista-galli. Phytochemistry 52, 373-382.

Marinova, K., Pourcel, L., Weder, B., Schwarz, M., Barron, D., Routaboul, J. M., Debeaujon, I., Klein, M., 2007. The Arabidopsis MATE transporter TT12 acts as a vacuolar flavonoid/ $\mathrm{H}^{+}$-antiporter active in proanthocyanidin-accumulating cells of the seed coat. Plant Cell 19, 2023-2038.

Maron, L. G., Pineros, M. A., Guimaraes, C. T., Magalhaes, J. V., Pleiman, J. K., Mao, C., Shaff, J., Belicuas, S. N., Kochian, L. V., 2010. Two functionally distinct members of the MATE (multi-drug and toxic compound extrusion) family of transporters potentially underlie two major aluminum tolerance QTLs in maize. Plant J 61, 728-740.

Mende, P., Wink, M., 1987. Uptake of the Quinolizidine Alkaloid Lupanine by Protoplasts and Isolated Vacuoles of Suspension-cultured Lupinus polyphyllus Cells. Diffusion or Carrier-mediatede Transport? J. Plant. Physiol. 129, 229-242.

Morita, M., Shitan, N., Sawada, K., Van Montagu, M. C., Inze, D., Rischer, H., Goossens, A., Oksman-Caldentey, K. M., Moriyama, Y., Yazaki, K., 2009. Vacuolar transport of nicotine is mediated by a multidrug and toxic compound extrusion (MATE) transporter in Nicotiana tabacum. Proc Natl Acad Sci U S A 106, 2447-2452.

Otani, M., Shitan, N., Sakai, K., Martinoia, E., Sato, F., Yazaki, K., 2005.

Characterization of vacuolar transport of the endogenous alkaloid berberine in Coptis japonica. Plant Physiol 138, 1939-1946.

Pighin, J. A., Zheng, H., Balakshin, L. J., Goodman, I. P., Western, T. L., Jetter, R., Kunst, L., Samuels, A. L., 2004. Plant cuticular lipid export requires an ABC transporter. Science 306, 702-704.

Sakai, K., Shitan, N., Sato, F., Ueda, K., Yazaki, K., 2002. Characterization of berberine transport into Coptis japonica cells and the involvement of $\mathrm{ABC}$ protein. J Exp Bot 53, 1879-1886.

Santelia, D., Vincenzetti, V., Azzarello, E., Bovet, L., Fukao, Y., Duchtig, P., Mancuso, S., Martinoia, E., Geisler, M., 2005. MDR-like ABC transporter AtPGP4 is involved in auxin-mediated lateral root and root hair development. FEBS Lett 579, 5399-5406. 
Sato, F., Yamada, Y., 1984. High berberine-producing cultures of Coptis japonica cells. Phytochemistry 23, 281-285.

Sato, H., Taguchi, G., Fukui, H., Tabata, M., 1992. Role of macil acid in solubilizing excess berberine accumulating in vacuoles of Coptis japonica. Phytochemistry 31 , 3451-3454.

Servos, J., Haase, E., Brendel, M., 1993. Gene SNQ2 of Saccharomyces cerevisiae, which confers resistance to 4-nitroquinoline- $N$-oxide and other chemicals, encodes a $169 \mathrm{kDa}$ protein homologous to ATP-dependent permeases. Mol Gen Genet 236, 214-218.

Shitan, N., Bazin, I., Dan, K., Obata, K., Kigawa, K., Ueda, K., Sato, F., Forestier, C., Yazaki, K., 2003. Involvement of CjMDR1, a plant multidrug-resistance-type ATP-binding cassette protein, in alkaloid transport in Coptis japonica. Proc Natl Acad Sci U S A 100, 751-756.

Shitan, N., Yazaki, K., 2007. Accumulation and membrane transport of plant alkaloids. Curr Pharm Biotechnol 8, 244-252.

Shoji, T., Inai, K., Yazaki, Y., Sato, Y., Takase, H., Shitan, N., Yazaki, K., Goto, Y., Toyooka, K., Matsuoka, K., Hashimoto, T., 2009. Multidrug and toxic compound extrusion-type transporters implicated in vacuolar sequestration of nicotine in tobacco roots. Plant Physiol 149, 708-718.

Terasaka, K., Blakeslee, J. J., Titapiwatanakun, B., Peer, W. A., Bandyopadhyay, A., Makam, S. N., Lee, O. R., Richards, E. L., Murphy, A. S., Sato, F., Yazaki, K., 2005. PGP4, an ATP binding cassette P-glycoprotein, catalyzes auxin transport in Arabidopsis thaliana roots. Plant Cell 17, 2922-2939.

Tommasini, R., Vogt, E., Fromenteau, M., Hortensteiner, S., Matile, P., Amrhein, N., Martinoia, E., 1998. An ABC-transporter of Arabidopsis thaliana has both glutathione-conjugate and chlorophyll catabolite transport activity. Plant J 13, 773-780.

Ukitsu, H., Kuromori, T., Toyooka, K., Goto, Y., Matsuoka, K., Sakuradani, E., Shimizu, S., Kamiya, A., Imura, Y., Yuguchi, M., Wada, T., Hirayama, T., Shinozaki, K., 2007. Cytological and biochemical analysis of COF1, an Arabidopsis mutant of an ABC transporter gene. Plant Cell Physiol 48, 1524-1533.

Verrier, P. J., Bird, D., Burla, B., Dassa, E., Forestier, C., Geisler, M., Klein, M., Kolukisaoglu, U., Lee, Y., Martinoia, E., Murphy, A., Rea, P. A., Samuels, L., Schulz, B., Spalding, E. J., Yazaki, K., Theodoulou, F. L., 2008. Plant ABC 
proteins--a unified nomenclature and updated inventory. Trends Plant Sci 13, 151-159.

Weid, M., Ziegler, J., Kutchan, T. M., 2004. The roles of latex and the vascular bundle in morphine biosynthesis in the opium poppy, Papaver somniferum. Proc Natl Acad Sci U S A 101, 13957-13962.

Yamada, S., Katsuhara, M., Kelly, W. B., Michalowski, C. B., Bohnert, H. J., 1995. A family of transcripts encoding water channel proteins: tissue-specific expression in the common ice plant. Plant Cell 7, 1129-1142.

Yazaki, K., 2005. Transporters of secondary metabolites. Curr Opin Plant Biol 8, 301-307.

Yazaki, K., 2006. ABC transporters involved in the transport of plant secondary metabolites. FEBS Lett 580, 1183-1191.

Yazaki, K., Kunihisa, M., Fujisaki, T., Sato, F., 2002. Geranyl diphosphate:4-hydroxybenzoate geranyltransferase from Lithospermum erythrorhizon. Cloning and characterization of a ket enzyme in shikonin biosynthesis. J Biol Chem 277, 6240-6246.

Yazaki, K., Shitan, N., Sugiyama, A., Takanashi, K., 2009. Cell and molecular biology of ATP-binding cassette proteins in plants. Int Rev Cell Mol Biol 276, 263-299.

Yazaki, K., Shitan, N., Takamatsu, H., Ueda, K., Sato, F., 2001. A novel Coptis japonica multidrug-resistant protein preferentially expressed in the alkaloid-accumulating rhizome. J Exp Bot 52, 877-879.

Yazaki, K., Sugiyama, A., Morita, M., Shitan, N., 2008. Secondary transport as an efficient membrane transport mechanism for plant secondary metabolites Phytochemistry Reviews 7, 513-524.

Zenk, M. H., Juenger, M., 2007. Evolution and current status of the phytochemistry of nitrogenous compounds. Phytochemistry 68, 2757-2772. 


\section{Figure Legends}

Fig. 1. Phylogenetic tree of the plant ABCB-type ABC transporters

The unrooted phylogenetic tree is based on a multiple alignment of the peptide sequences of ABCBs from Arabidopsis, wheat, barley, potato, upland cotton, and Coptis japonica, produced by the ClustalW program (http://align.genome.jp/). The resultant tree was produced using TREEVIEW. Arabidopsis ABCB sequences were recovered according to the AGI-Code described by Verrier et al. (2008). Accession numbers BAB85651, BAB62040, AAF23176, AAD10836, CAA71179, AAR00316, and AAR10387, correspond to the transporters, TaMDR1 (wheat, Triticum aestivum), CjABCB1 (Coptis japonica), CMDR1 (cotton, Gossypium hirsutum), PMDR1 (potato, Soalnum tuberosum), HvMDR2 (barley, Hordeum vulgare), ZmPGP1 (maize, Zea mays), dw3 (Sorghum bicolor), respectively.

Fig. 2. Northern hybridization of $C j a b c b 2$ and 3 (A), and berberine accumulation in cultured $C$. japonica cells (B)

The cultured cells were harvested 2, 5, 9, 14, 21 days after inoculation, $3 \mu \mathrm{g}$ of poly $(\mathrm{A})^{+}$RNA was isolated and loaded on formamide-containing agarose gel. As the hybridization probe, 3'-untranslated region of Cjabcb2 (200 bp) and Cjabcb3 (200 bp) were used. ATP synthase ( $\beta$ subunit) was used as the load control. Berberine content in the cells was monitored by HPLC analysis.

Fig. 3. Northern analysis of Cjabcb2 in intact $C$. japonica plant 
Organ-specific expression of $C j A B C B 2$ in the intact plant and cultured cells of $C$. japonica detected by Northern hybridization (probe: 200 bp fragment of Cjabcb2 3'-untranslated region). Total RNA (10 $\mu \mathrm{g})$ was isolated from each organ and separated on formamide-containing agarose gel. As the load control, 18S rRNA was used.

Fig. 4. Functional characterization of $\mathrm{Cj} A B C B 2$ using yeast cells Yeast strain AD12345678 was transformed with pDR196 (empty vector) or pDR-CjABCB2. Each transformant was pre-cultured overnight in SD medium (-uracil). The cultures were diluted to A600=0.5. Five $\mu 1$ was spotted onto an SD medium (-uracil) plate containing Ber (berberine, $3.5 \mu \mathrm{M}$ ), 4-NQO (4-nitroquinoline $N$-oxide, $0.11 \mu \mathrm{g} / \mathrm{ml}$ ) or Cyc (cycloheximide, $0.0175 \mu \mathrm{g} / \mathrm{ml}$ ) and incubated for $44 \mathrm{~h}$ at $25^{\circ} \mathrm{C}$.

Fig. 5. Plasma membrane localization of $\mathrm{Cj} \mathrm{ABCB} 2$ in $C$. japonica cells (A) Microsomal fraction proteins $(40 \mu \mathrm{g})$ of $C$. japonica cells and membrane proteins (20 $\mu$ g per lane) of yeast cells with pDR196 (vector control), or pDR196-CjABCB2 were separated by SDS-PAGE and blotted onto a PVDF membrane. The membrane was probed with anti-CjABCB2 polyclonal antibodies.

(B) Immuno-detection of $\mathrm{Cj} \mathrm{ABCB} 2$ in $C$. japonica plasma membranes. Fractionation of total microsomes from $C$. japonica cells on a non-continuous sucrose gradient consisting of $0,20,30,40$, and 50\% (wt/vol) sucrose. Membrane fractions from the interfaces between different sucrose concentrations were collected and immuno-detected with anti-CjABCB2 antibodies. Blots were probed with antibodies raised against $\mathrm{Cj} A B C B 2$, plasma membrane $\mathrm{H}+$-ATPase $\left(\mathrm{H}^{+}\right.$-ATPase $)$, vacuolar $\mathrm{H}^{+}$-pyrophosphatase (V-PPase), and endoplasmic reticulum BiP (BiP). 
(C) Microsomal membranes (Mic) from C. japonica cells were fractionated by the aqueous two-phase partitioning method into an upper phase (U) enriched with plasma membranes and a lower phase (L) containing intracellular membranes. Proteins from each fraction (10 $\mu \mathrm{g}$ per lane) were immuno-blotted. The same membrane was re-probed with antibodies against the indicated marker proteins.

Fig. 6. In situ hybridization of $C j a b c b 2$ in the rhizome and root of $C$. japonica (A, B) In situ hybridization using a digoxigenin-labeled antisense (A) and sense (B) probe in the rhizome. (C, D) In situ hybridization using a digoxigenin-labeled antisense (C) and sense (D) probe in the root. BF, bast fiber; C, cortex; E, epidermis; M, medulla; Ph, phloem; VB, vascular bundle; X, xylem. Bars, 500 mm. 
Clade I

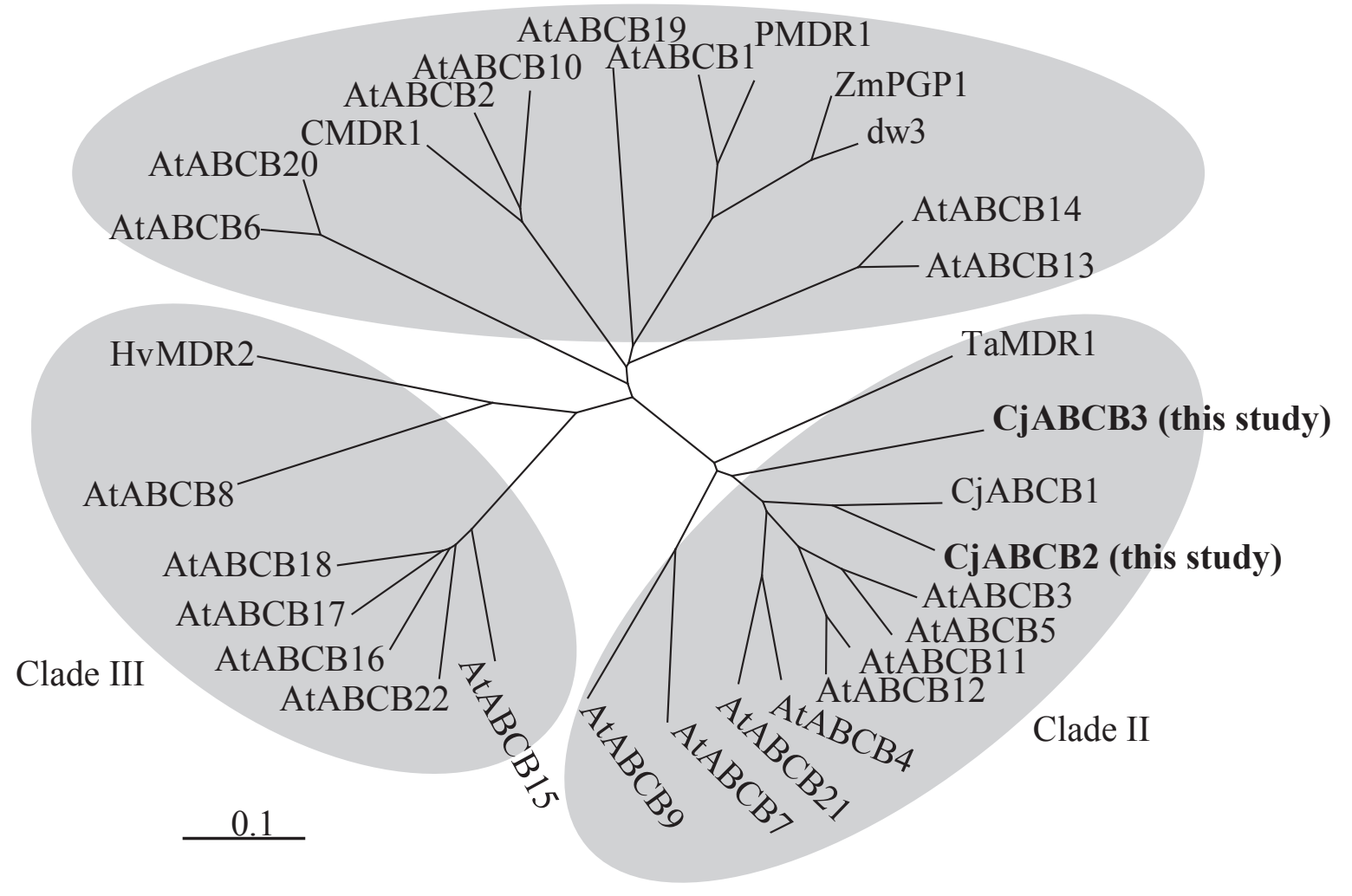

Shitan et al. Fig. 1 
(A) culture period (days)

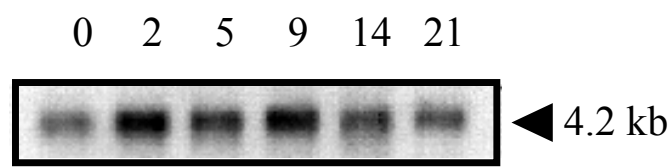

Cjabcb2

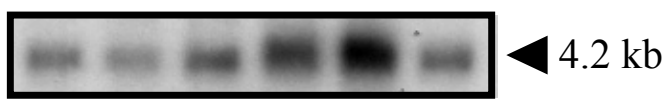

Cjabcb3

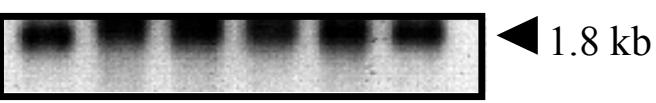

(B)

ATP synthase $(\beta)$

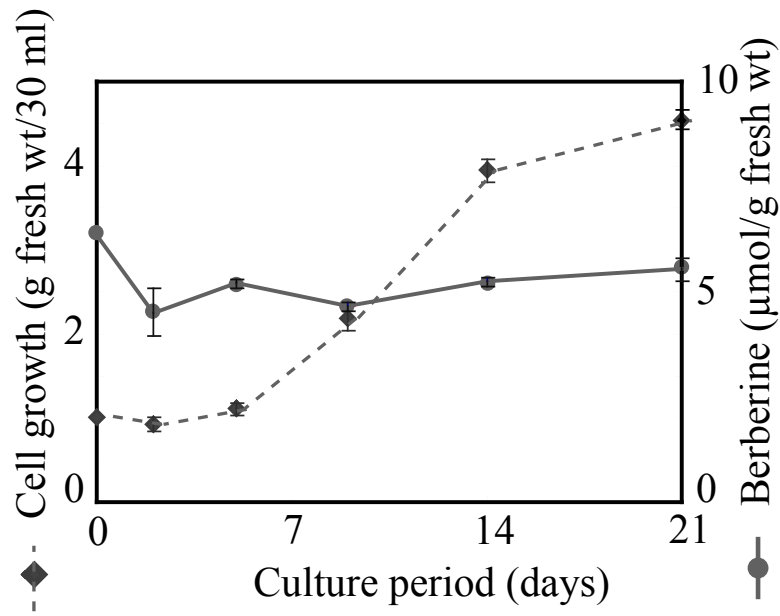

Shitan et al. Fig. 2 


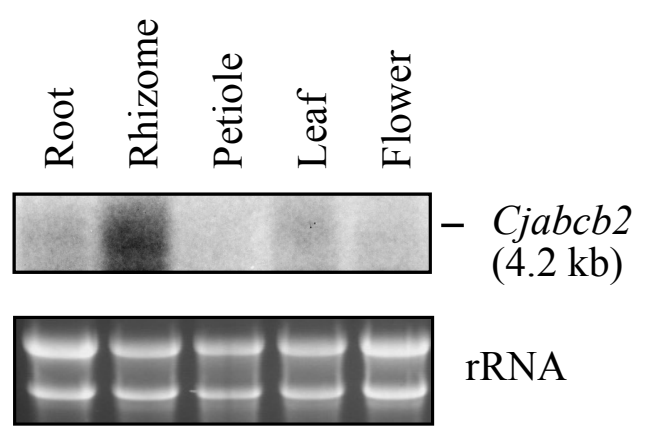

Shitan et al. Fig. 3 


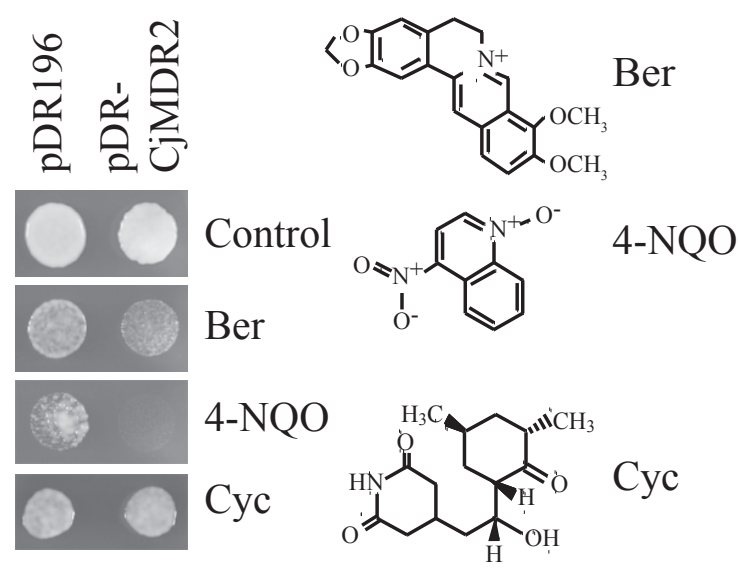

Shitan et al. Fig. 4 


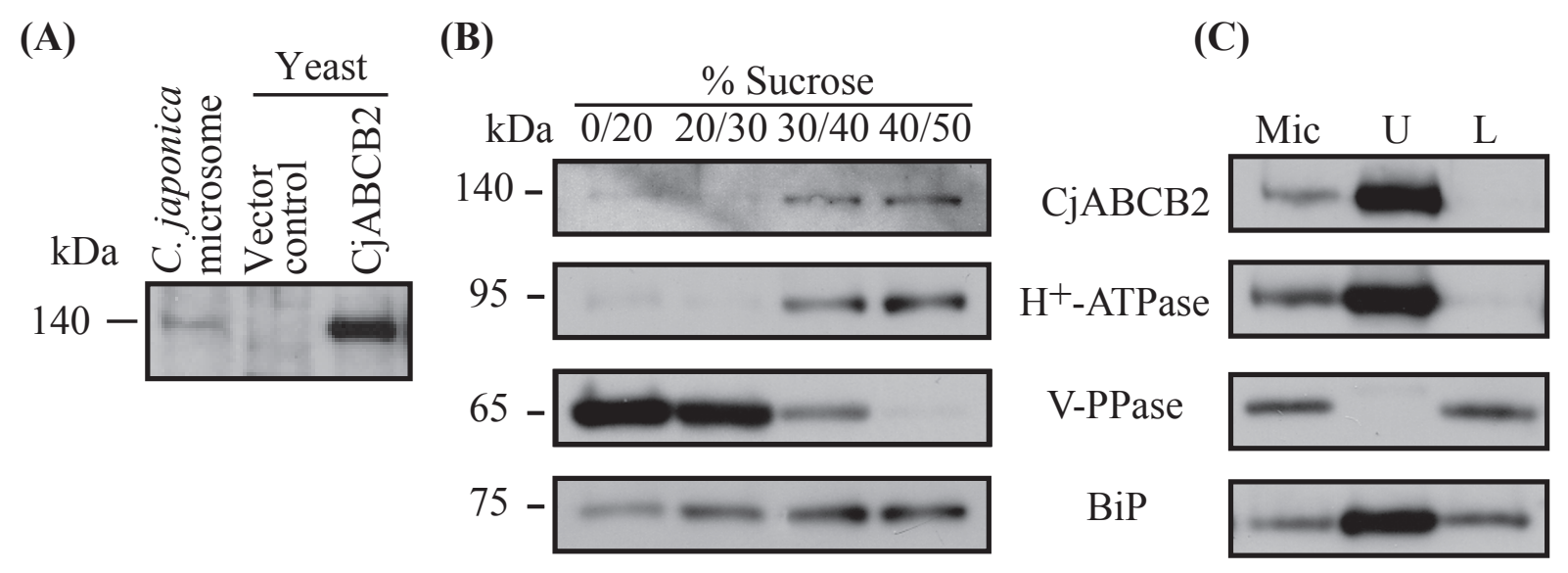

Shitan et al. Fig. 5 
A

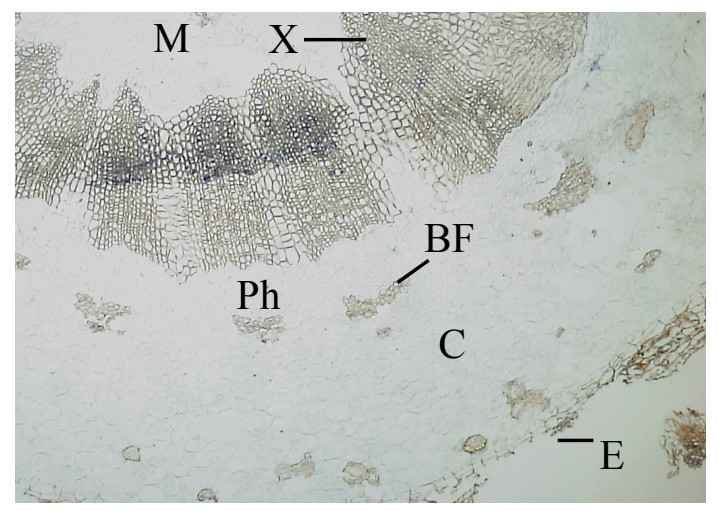

C

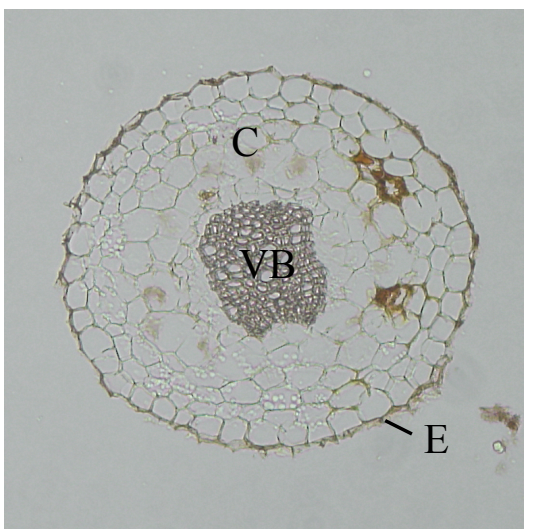

D
B
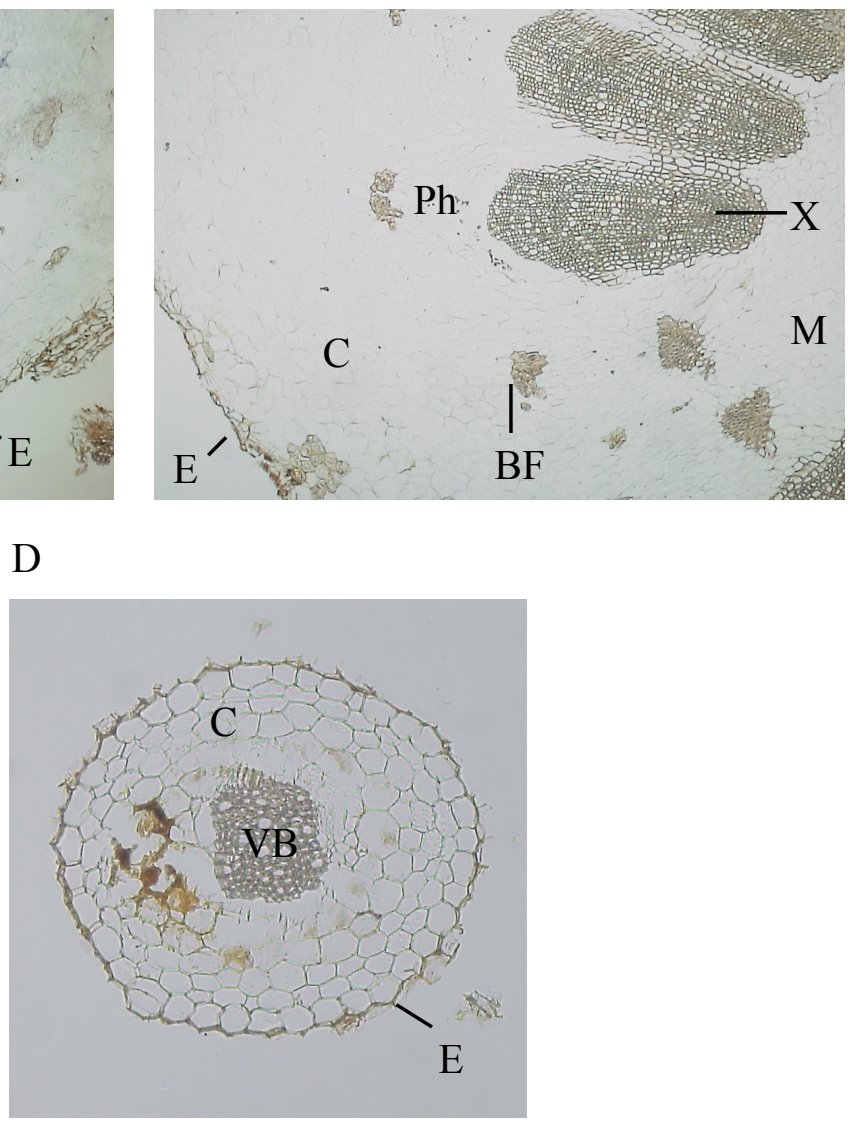

Shitan et al. Fig. 6 